\title{
Glass Surface Defects Detection with Wavelet Transforms
}

\author{
Bayram Akdemir and Şaban Öztürk
}

\begin{abstract}
Nowadays, the increasing population increases the consumption as well. Manufacturing systems are developing in a fast pace to meet increasing demand of consumption. However, very quick increase of production has surpassed the development speed of currently existing control systems. In manufacturing, since the quality is a very important issue as well as the quantity, the operation of quality control systems must be accelerated and must be accomplished by machines. The idea of our study is based on this thinking. Especially defect detection on widely used glass material that is extremely difficult to accomplish by humans can be implemented in a quick, accurate and stable way. In the presented method, various defects like scratches, bubbles, cracks, corrosion on the glass surface can be identified. Glass images obtained from a homogenously illuminated medium are processed by wavelet transform and obtained images from the wavelet transform are denoised. Finally, Shannon threshold method is applied to the images. From the obtained results, defects like scratches, bubbles on the surface of the glass can be detected successfully.
\end{abstract}

Index Terms - Glass defect detection, texture analysis, image processing, wavelet transform, machine vision.

\section{INTRODUCTION}

Nowadays, as a result of the rapidly expanding automation systems, it is possible to increase number of manufactured products in the proportion of supply and demand. In the globalized world, very fast and effective productions in low cost are aimed to be prepared for possible demands. Depending on the arbitrary or compulsory requirements, glass and glass products are preferred in many different sectors such as packaging, mirror, kitchen industry, insulation, heating. Among the manufactured glass products, defected ones must be prevented to reach to the customer. To ensure that, the manufactured products must be quality controlled before leaving the factory. Quality control after production is usually made by a machine network included to the manufacturing automation to reduce defects. Machine-based quality control systems are quite advantageous compared to the human-based ones [1]-[3]. However, during the search for possible defects on the glass by sensory technologies, the transparency and light transmittance of the glass lead to extreme difficulties and long delays to obtain desired results [4], [5]. For this, the workload of camera which is image acquisition sensor should be facilitated. One of the most important points is lighting system. The lighting system must be designed taking into account features of the camera. Lighting system should be effectively illuminated the glass surface. Its main task is to

Manuscript received December 4, 2014; revised March 31, 2015. This project (114E925) is supported by TUBITAK.

The authors are with the Electrical and Electronics Engineering Department, Engineering Faculty, University of Selcuk, Konya, Turkey (e-mail: \{bayakdemir, sabanozturk\}@ selcuk.edu.tr). help image processing unit through illuminating homogeneously whole glass surface. Other important issue is position of the camera. It must be correctly calculate size of defects on the glass surface. Thus, one or more cameras can be used. If the angle increases between the glass surface and the camera, calculations and estimating shape of defects becomes more difficult.

Possible problems encountered on the glass during production are defects like scratches, bubbles, cracks and fractures. When these problems are detected during quality control procedure, the products can be recycled and reprocessed before they leave the factory. However, if the defected glass reaches the consumer, besides increasing the cost of recycling, the factory may be discredited. Therefore, it is clear that the quality control must be made very carefully and the product must be presented to the customer after this. In this study, by implementing the quality control of glass in an effective way, an effort is made to increase the quality of the manufactured glass and avoid possible financial losses. A number of different methods have been employed in glass defect analysis and many studies for effective control are present in literature. In order to detect the surface defects on the glass, Markov random field [6] Otsu threshold method [7], canny edge detection method [8], binary feature histogram method [9], Fuzzy C Means clustering algorithm [10] have been employed. In this study, the images taken from homogenously illuminated medium are first preprocessed to be denoised, processed via wavelet transform and transformed into binary form by Shannon method. Shannon Method is a common method to evaluate the edges [11]-[13]. Therefore, the defects over the glass can be detected in a quick and accurate manner.

Wavelet analysis is becoming a popular tool for analyzing localized variations of power within a time series. By decomposing a time series into time-frequency space, one is able to determine both the dominant modes of variability and how those modes vary in time and wavelet transform has been used for numerous studies such as image processing, geophysics, ocean waves etc. [14]-[16].

In this study, the taken images have been evaluated using wavelet transform for surface detection. Wavelet improves Shannon entropy evaluates. Wavelet and Shannon entropy were used together to determine the edges.

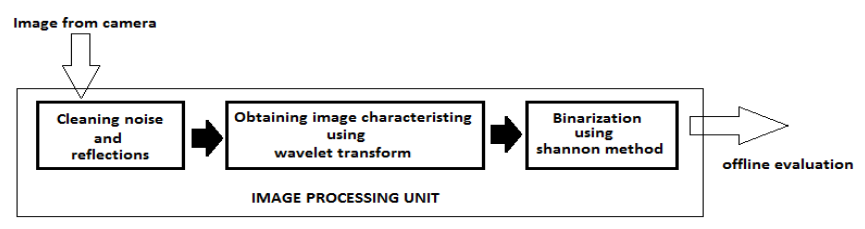

Fig. 1. Image processing unit.

Rest of this paper is organized as follow; Section II is mentioned about system overview and definition of problem. Section III is explained wavelet transform and using this 
paper. Section IV, basic principles of Shannon entropy theorem are introduced. At Section V, proposed system is tested and test results are presented. And finally. Section VI contains conclusion.

\section{SYSTEM OVERVIEW}

Due to its structure, glass is very prone to reflections. These reflections complicate examine the glass surface. Therefore, during the identification of glass defect by camera, ambient lighting conditions are extremely important. In case the ambient lighting is made improperly, camera may not be able to detect defects on the glass. The important issues in lighting is that the source cannot be concentrated at one point i.e. it must be homogenous and must be applied to detect glass defects in the most effective way. There are significant advantages of homogeneous illumination. Some of them; can be analyzed using more simple image processing algorithms, can be a cheaper system than other systems, glass reflections is partially prevented and thus background noises are removed easier. The lighting made this way can facilitate the task of detect analysis. The homogenously illuminated images obtained by a camera is subjected to an image processing mechanism as shown in Fig. 1. Here, first of all, the image is taken from the homogenously illuminated medium by a camera. One camera is used to capture images. Camera is placed in front of the glass surface. So, surface images is captured two-dimensional. As the depths of defects are not search, 2-D images are more useful for this work. Depending on the lighting of the medium, the images taken include some noise due to the reflection from the glass. These noises can be removed by image pre-processing. Image pre-processing algorithms are generally very simple and it is used for simple operations. Elimination of noise in the glass images is not simple. Because the algorithms used to eliminate noises evaluated as a noise in tiny defects on the glass surface. When the glass surface noises are cleaned using this simple algorithms, tiny defects in the glass surface are removed from the image. Incorrect results are obtained in such a measurement. So, more specific methods are used to remove noises in the glass surface inspection process. In this work, reference image system is used. Working principle of this system is as follow; firstly perfect glass image is capture, then determined noises on this glass. As long as measurement medium is not changed, the noises are the same on all glass. Therefore, the noises which are obtained from perfect glass is removed from all analyzed glass. After noises cleaned, image characteristics are analyzed.

Afterwards, in order to unveil the quality of the image, two-dimensional wavelet analysis is used. Binarization of the images obtained from wavelet analysis is accomplished by Shannon threshold method. To purpose of making binarization make more meaningful the image.

\section{WAVELET TRANSFORM}

Wavelet analysis in its present theoretical form has been first introduced by Jean Morlet. Wavelets process the received signal by separating it into its constituent frequencies. The main idea here is that by separating the incoming signal into its sub-components, it can be observed both in time and frequency domain by suitable wavelets. Processing both in time and frequency domain is the main property of wavelet transform. In the analysis of high frequency signals, wavelet transform must be designed in such a way that it gives a good time resolution and poor frequency resolution [17], [18]. Otherwise, in case low frequency signals are involved, it must be designed to yield a good frequency resolution and a poor time resolution. Wavelet is a common method to evaluate the signals [19], [20]. $\Psi(\mathrm{t})$ represents the fundamental wavelet and it can be calculated as given in (1) and (2).

$$
\begin{gathered}
\psi_{(S, \zeta)}(t)=\frac{1}{\sqrt{s}} \psi \frac{t-r}{S} \\
\Upsilon(S, \zeta)=\int f(t) \Psi^{t}(s, \zeta) t(d t)
\end{gathered}
$$

In recent years, wavelet transform methods have started to be widely used. In particular, in the field of image processing, they have found increasingly widespread use for the purposes of image compression, evaluation of radar images, surface analysis, computer vision systems, just to name a few.

\section{SHANNON ENTROPY THEOREM}

In Shannon entropy method, the values a case or an event can get in case of random conditions are defined mathematically. Among numerous application fields of Shannon entropy method, in the proposed study it will be employed for the purpose of image segmentation that is a stage of image processing [21]-[23]. In this usage, the main purpose is to optimally segment the image using a threshold value obtained in regard to the calculated probability values considering the pixel values in the image. Let ' $a$ ' be the image taken from the real-world. First of all, ' $a$ ' is converted to a gray-level image and each pixel in the image is assigned a value from 0 to 255 . Afterwards, the histogram of gray-level image is calculated and the condition of each pixel is considered in probability calculation. Equation (3) shows the calculation of Shannon entropy value.

$$
H_{(a)}=\sum_{i} P_{i} \log _{2} \frac{1}{P_{i}}=-\sum_{i} P_{i} \log _{2} P_{i}
$$

Finally, the optimal threshold value is determined and the image is binarized. During this procedure, the data is divided into two distinct classes based on determined threshold value. Upper than threshold level data value turns to 255 and under the threshold data turns to 0. Only two possibilities occur as black or white. Binary thresholding is accomplished to complete binary image segmentation process.

\section{EXPERIMENTAL RESULTS}

In this study, 16 glasses analysis is performed. Some of these glasses are scratch, some of these have bubbles, some of these are cracks etc. and 3 glasses are perfect. The 
dimension of glasses are $12 \times 18 \mathrm{~cm}$. There are two types of defects in inspected glasses. These; production defects and defects generated by us. As the glass is a material prone to reflections, extreme care must be given to the lighting system and to the medium the image is taken during the glass surface analysis by a camera. The images are taken by a camera from a medium illuminated in a controlled and homogenous manner exposed to the minimum ambient noise. The camera used is a low resolution one (1.2 MP, $1024 \times 768)$. The images taken are processed in regard to the following three stages.

Step 1: The surface properties of the images taken from the camera are observed by wavelet transform and their attributes related with the defects are extracted.

Step 2: Glass reflections and camera's status information is extracted from the images having foregrounded surface attributes. At the same time, the likely occurred noise is eliminated using average filter.

Step 3: In the last step, the images are segmented using Shannon entropy method. In segmentation method, in order to detect the defected zones more easily, binary thresholding has been used. Therefore, the defected zones will be in completely opposite color to be detected easily.

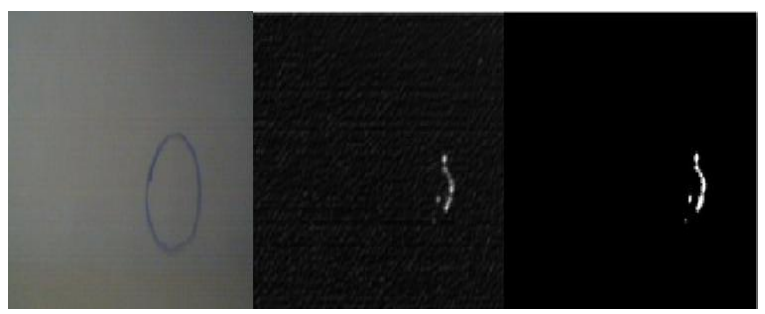

(a)

(b)

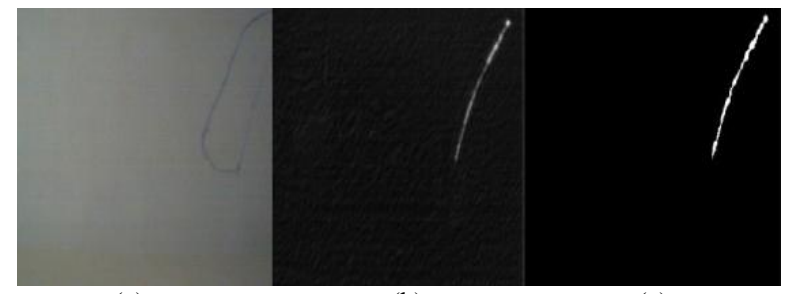

(a)

(b)

(c)

Fig. 2. Evaluated pictures: (a) Scratch on the transparency glass. (b) After wavelet evaluation. (c) Shannon method improves the clearance of the scratches on the picture.

The identified glass is shown in the leftmost image in Fig. 2. It is extremely difficult to detect the defected zone by eye. Particularly, considering an expert spending hours to make similar observations, it is clear that an erroneous decision is highly possible. In Fig. 2, the images in the middle of Fig. 2 show the ones, in which their attributes are extracted using Wavelet analysis, and then denoised to obtain clean images. Shannon method has been applied to these images to implement thresholding. In the experiments results, vast majority of defect in the glass are determined. But some tiny defects are not determined. One reason for this is low quality of camera. Image details taken in low resolution can be missed. In noise reduction process, the classical methods evaluate tiny defects as noise. In the reference image noise reduction methods vary depending on the glass surface reflections. So the results of the analysis has a fault tolerance. To reduce error tolerance, a noise reduction method which is designed according to the properties of material should be used. In the noise reduction method designed in this study tiny defects are perceived as a noise due to different curvature of glass surface. And this defects are subtracted from image.

\section{CONCLUSION}

Proposed method aims to find glass defects on the transparency surface based on wavelet and Shannon binarization methods. The proposed system has been investigated in the analysis of flat glasses and very promising results have been obtained. The results obtained from this study is more reliable compared to the analysis made by human eye. Because the human eyes are tired with times, successful rate is lower. In the examination of the machine, error rate does not change over time. Proposed system is very cheap and simple. Considering the detection speed of the performed method, it can be eligible to include to the manufacturing system of the factory. Besides, owing to the flexibility of the proposed image processing algorithm, defect detection procedure can be applied not only to the glass surface but also to the surfaces of fabric, aluminum, iron plates and tiles. Glass defect inspection time is around millisecond. The distance between the camera and the glass can be changed. Thus, different sizes of glasses can be measured. Further studies will be carried out efforts for the detection of small defects. We will explore methods that can be used to reduce the error tolerance of the system. Glass defects will be inspected 3D using advanced cameras. Therefore, depth of defects can be calculated.

\section{REFERENCES}

[1] E. N. Malamas, E. G. Petrakis, M. Zervakis, L. Petit, and J. D. Legat, "A survey on industrial vision systems, applications and tools," Image and Vision Computing, vol. 21, no. 2, pp. 171-188, 2003.

[2] A. M. Zaid, S. A. Aziz, and M. Abdul, "Quality inspection of bakery production using a color-based machine vision system," Journal of Food Quality, vol. 23, no. 1, pp. 39-50, 2000

[3] J. Kaartinen, J. Hätönen, H. Hyötyniemi, and J. Miettunen, "Machine-vision-based control of zinc flotation - A case study," Control Engineering Practice, vol. 14, no. 12, pp. 1455-1466, 2006.

[4] M. Domingo and M. Olaya, "Automated visual inspection of glass bottles using adapted median filtering," Image Analysis and Recognition, Springer Berlin Heidelberg, vol. 3212, pp. 818-825, 2004.

[5] A. Francesco, "An Automated visual inspection system for the glass industry," in Proc. the 16th IMEKO TC4 Symposium, Florence, Italy, 2008, vol. 9.

[6] J. Ai and X. Zhu, "Analysis and detection of ceramic-glass surface defects based on computer vision," in Proc. the 4th World Congress on Intelligent Control and Automation, 2002, vol. 4, pp. 3014-3018.

[7] X. Peng, Y. Chen, W. Yu, Z. Zhou, and G. Sun, "An online defects inspection method for float glass fabrication based on machine vision," The International Journal of Advanced Manufacturing Technology, vol. 39, issue 11-12, pp. 1180-1189, 2008.

[8] F. Adamo, F. Attivissimo, A. Di Nisio, and M. Savino, "An online defects inspection system for satin glass based on machine vision," in Proc. the Instrumentation and Measurement Technology Conference, I2MTC'09 IEEE, 2009, pp. 288-293.

[9] J. Zhao, Q. J. Kong, X. Zhao, J. Liu, and Y. Liu, "August. A method for detection and classification of glass defects in low resolution images," in Proc. the 2011 Sixth International Conference on Image and Graphics (ICIG), 2011, pp. 642-647.

[10] J. George, S. Janardhana, J. Jaya, and K. J. Sabareesaan, "Automatic defect detection inspectacles and glass bottles based on fuzzy $\mathrm{C}$ means clustering," in Proc. the 2013 International Conference on Current Trends in Engineering and Technology, 2013, vol. 2, pp. 8-12.

[11] B. Singh and A. P. Singh, "Edge detection in gray level images based on the Shannon entropy," Journal of Computer Science, vol. 4, no. 3 , pp. 186, 2008. 
[12] Z. J. Hou and G. W. Wei, “A new approach to edge detection," Pattern Recognition, vol. 35, no. 7, pp. 1559-1570, 2002.

[13] Y. J. Yu and S. T. Acton, "Edge detection in ultrasound imagery using the instantaneous coefficient of variation," IEEE Transactions on Image Processing, vol. 13, no. 12, pp. 1640-1655, 2004.

[14] H. Y. Weng and K. M. Lau, "Wavelets, period doubling, and time-frequency localization with application to organization of convection over the tropical western Pacific," Journal of the Atmospheric Sciences, vol. 51, no. 17, pp. 2523-2541, 1994.

[15] S. R. Massel, "Wavelet analysis for processing of ocean surface wave records," Ocean Engineering, vol. 28, no. 8, pp. 957-987, 2001.

[16] H. H. Tong, "Blur detection for digital images using wavelet transform," in Proc. the 2004 IEEE International Conference on Multimedia and Expo, 2004, vol. 1.

[17] A. Graps, "An introduction to wavelets," Computational Science \& Engineering, vol. 2, pp. 50-61, 1995.

[18] O. Rioul and M. Vetterli, "Wavelets and signal processing," IEEE Signal Processing Magazine, vol. 8, issue 4, pp. 14-38, 1991.

[19] V. J. Samar, "Wavelet analysis of neuroelectric waveforms: A conceptual tutorial," Brain and Language, vol. 66, no. 1, pp. 7-60, 1999.

[20] S. T. Quek, "Sensitivity analysis of crack detection in beams by wavelet technique," International Journal of Mechanical Sciences, vol. 43, no. 12, pp. 2899-2910, 2001.

[21] C. E. Shannon, "A mathematical theory of communication," $A C M$ SIGMOBILE Mobile Computing and Communications Review, vol. 5 , no. 1, pp. 3-55, 2001

[22] R. M. Gray, Entropy and Information Theory, vol. 1. New York: Springer, 2011
[23] R. R. Coifman and M. V. Wickerhauser, "Entropy-based algorithms for best basis selection," IEEE Transactions on Information Theory, vol. 38 , no. 2, pp. 713-718, 1992

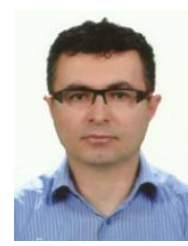

Bayram Akdemir was born in Konya, Turkey in 1974 He received the B.S. degree in electrical \& electronics engineering from Selçuk University, Turkey, in 1999, and the M.S. and Ph.D. degrees in electrical \& electronics engineering from Selçuk University Konya, Turkey, in 2004 and 2009, respectively.

In 1999, he joined the Department of Electrical \& Electronics Engineering, Selçuk University, as a research assistant. Currently, he is an assistant professor in the same department. His current research interests include electronic circuits, sensors, artificial intelligence, renewable energy sources.

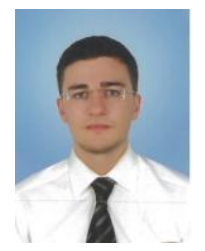

Şaban Öztürk was born in İzmir, Turkey in 1989. He received the B.S. degree in electronics education from Pamukkale University, Denizli, Turkey, in 2011. She continues to study M.S. degree in electrical \& electronics engineering in Selçuk University, Konya, Turkey.

Currently, he is a research assistant with the Electrical-Electronics Engineering Department, Selçuk University, Konya, Turkey. His current research interests include image processing, machine vision, computer vision, intelligent control and embedded systems. 